\title{
Plasma grid design for optimized filter field configuration for the NBI test facility ELISE
}

\author{
R. Nocentini*, R. Gutser, B. Heinemann, M. Froeschle, R. Riedl and the NNBI- \\ Team
}

Max-Planck-Institut für Plasmaphysik, EURATOM Association, D-85740 Garching, Germany

*Corresponding author. Tel.: +49 893299 1490, Fax: +49 893299 2558, e-mail: riccardo.nocentini@ipp.mpg.de

\begin{abstract}
Maintenance-free RF sources for negative hydrogen ions with moderate extraction areas (100-200 $\left.\mathrm{cm}^{2}\right)$ have been successfully developed in the last years at IPP Garching in the test facilities BATMAN and MANITU. A facility with larger extraction area $\left(1000 \mathrm{~cm}^{2}\right)$, ELISE, is being designed with a "half-size" ITER-like extraction system, pulsed ion acceleration up to $60 \mathrm{kV}$ for $10 \mathrm{~s}$ and plasma generation up to $1 \mathrm{~h}$. Due to the large size of the source, the magnetic filter field (FF) cannot be produced solely by permanent magnets. Therefore, an additional magnetic field produced by current flowing through the plasma grid (PG current) is required. The filter field homogeneity and the interaction with the electron suppression magnetic field have been studied in detail by finite element method (FEM) during the ELISE design phase. Significant improvements regarding the field homogeneity have been introduced compared to the ITER reference design. Also, for the same PG current a 50\% higher field in front of the grid has been achieved by optimizing the plasma grid geometry. Hollow spaces have been introduced in the plasma grid for a more homogeneous PG current distribution. The introduction of hollow spaces also allows the insertion of permanent magnets in the plasma grid.
\end{abstract}

Keywords: Negative ions; RF source; Filter field; Plasma grid; ELISE test bed; ITER NBI

\section{Introduction}

The neutral beam injection system for ITER requires a deuterium beam with an energy of $1 \mathrm{MeV}$ for up to $1 \mathrm{~h}$. In order to inject the required power of $17 \mathrm{MW}$ into the plasma, the ion source has to deliver $40 \mathrm{~A}$ of negative ion current. To develop the ion source a facility with large extraction area, ELISE [1], is being designed at IPP Garching with a "half-size" ITER-like extraction system, pulsed ion acceleration up to $60 \mathrm{kV}$ for $10 \mathrm{~s}$ and plasma generation up to $1 \mathrm{~h}$. The 
ELISE ion source is equipped with three grids that form the extraction system: plasma grid (PG), extraction grid (EG) and grounded grid (GG). The grids system accelerates the ion beam horizontally, in $Z$ direction. We refer to the vertical direction as $\mathrm{Y}$ direction and to the horizontal direction parallel to the grids as $\mathrm{X}$ direction.

A horizontal magnetic filter field (FF) is required in the extraction region. The FF permits to reduce the amount of fast electrons coming from the drivers that can detach the additional electron from the $\mathrm{H}$ - and neutralize them [2].

In large ion sources the FF can be produced by an electric current flowing vertically in the PG (PG current) and by additional permanent magnets positioned on the side of the source vessel. Other possibilities are under consideration.

Test on the FF produced by a PG current have already been carried out in the RADI facility [3]. While RADI allows a localized extraction of negative ions, ELISE will allow extraction from a large area grid, therefore it will be possible to study the effect of the FF on the homogeneity of large beams.

Calculations presented in this paper show that the PG current in the ITER reference design creates a non-homogeneous field in front of the PG. The PG of ELISE has been designed to minimize this non-homogeneity.

As the extraction system of ELISE has the same width as that of the ITER RF source, all the considerations valid for ELISE are also relevant for ITER.

\section{The ELISE PG geometry}


The ELISE PG geometry is compatible with the ITER reference design for the negative ion source [4]. The grid consists of two segments (four in ITER) made of copper. In each segment the apertures are arranged in beamlet groups (see fig. 1) to match the sub-division of the beam line components in the ITER neutral beam injector.

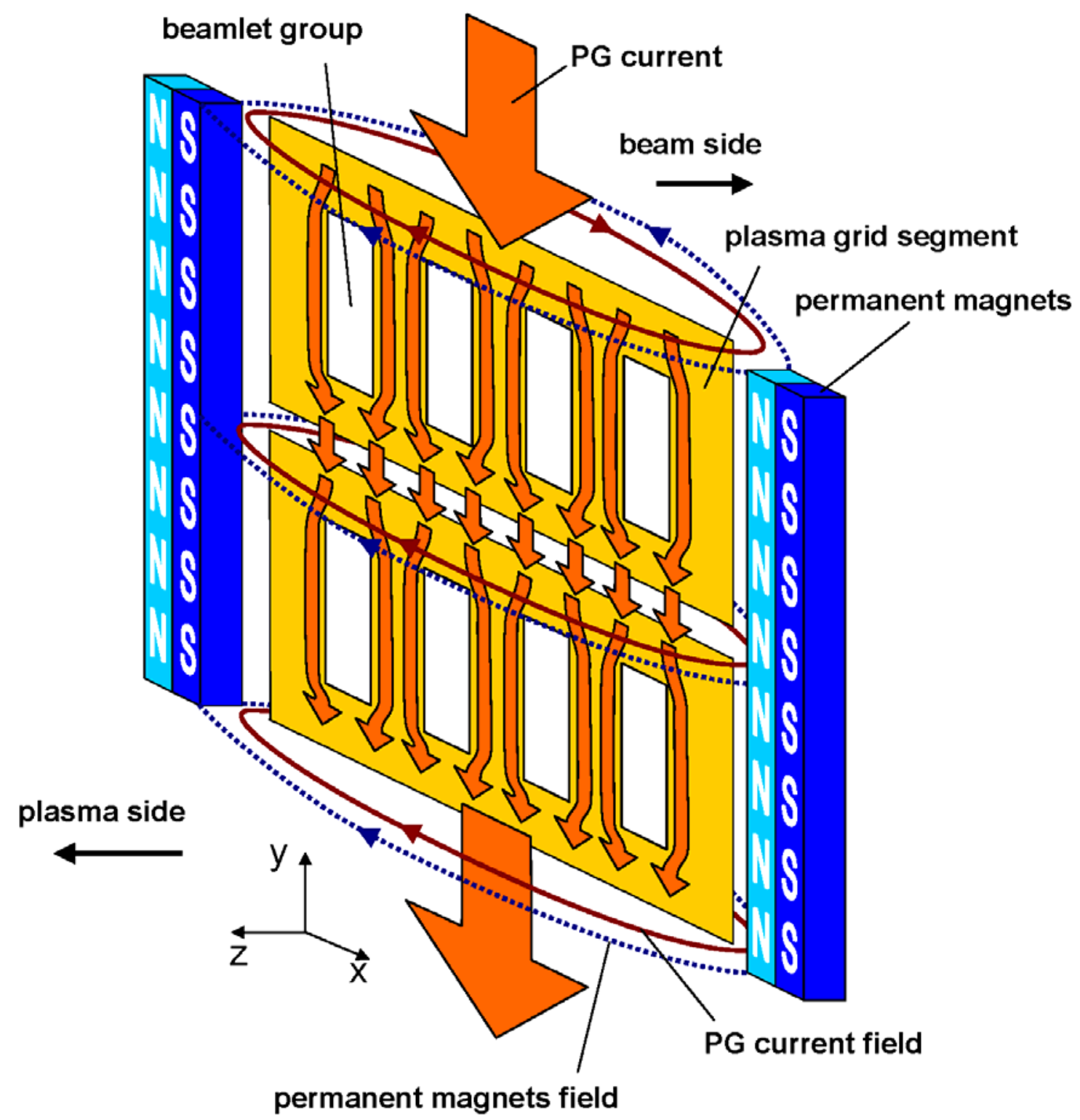

Figure 1 Magnetic FF scheme. In ITER the PG current flows mainly in between the beamlet groups and on the sides of the grid. PG current field and permanent magnets field add on the plasma side and subtract on the beam side. 
Each beamlet group is made of $16 \times 5$ apertures, with $20 \mathrm{~mm}$ spacing both in horizontal and vertical direction. The aperture diameter is $14 \mathrm{~mm}$.

On the basis of the IPP experience the profile of the apertures has been modified. The apertures have been shaped with an $80^{\circ}$ chamfer on the plasma side. In addition the original $6 \mathrm{~mm}$ thickness in the ITER reference design for the PG has been increased to $9 \mathrm{~mm}$. These two modifications increase the area for the conversion of neutrals and positive ions to negative in the region close to the apertures and allow a more suitable starting angle of the negative ions [2]. In ELISE the PG current is fed by means of 24 copper stripes attached to the back of the PG. While one end of these stripes is heated to $150{ }^{\circ} \mathrm{C}$ by the plasma grid, the other end is cooled. The geometry of the stripes is optimized to reduce thermal losses both with and without plasma grid current flowing and to allow thermal expansion of the grid. Between the PG segments 42 flexible connections can carry the current and allow for $3 \mathrm{~mm}$ horizontal and $1 \mathrm{~mm}$ vertical displacement (see fig. 2). The components are designed to allow up to 8 kA of total current. The PG is electrically insulated from the supporting structure to concentrate the current on the grid and hence to achieve a higher magnetic field. 


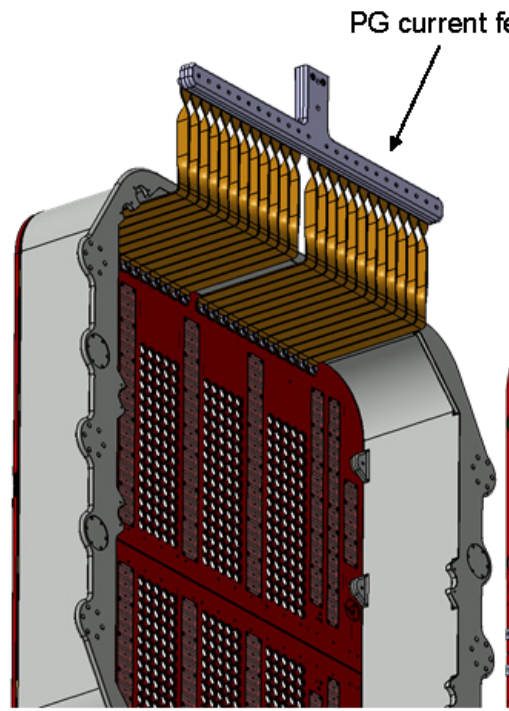

a)

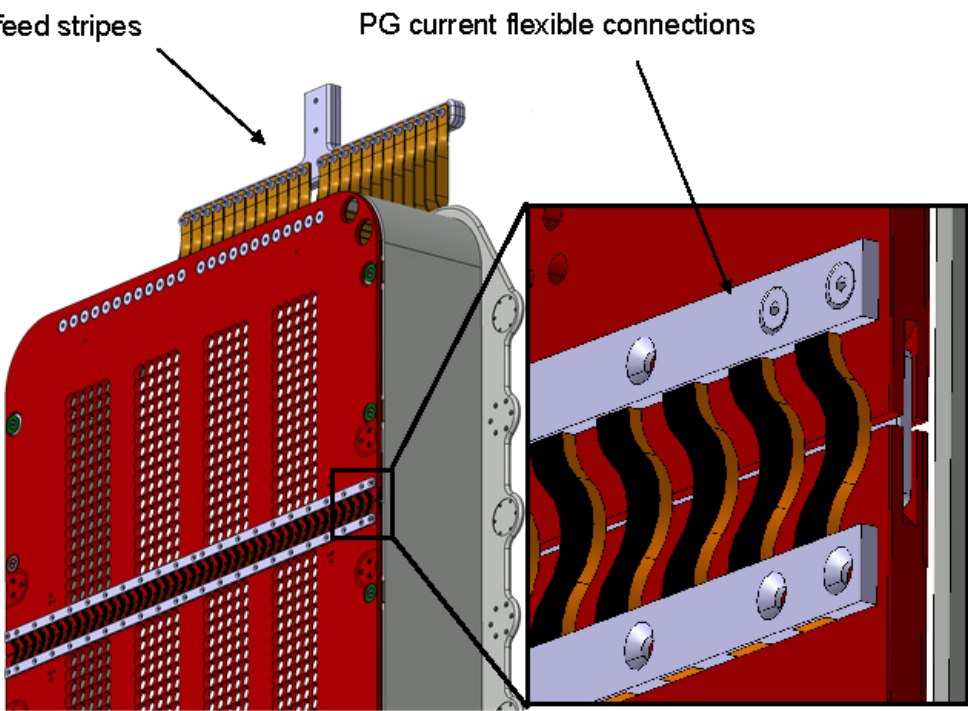

b)

Figure 2 (a) Plasma grid current feed with 24 copper stripes attached to the back of the plasma grid. On one end these stripes are heated to $150{ }^{\circ} \mathrm{C}$ by the plasma grid, the other end is cooled. Geometry is optimized to reduce thermal losses with and without plasma grid current. (b) Flexible connections between the plasma grid segments. 42 connections can carry $8 \mathrm{kA}$ total PG current and allow for $3 \mathrm{~mm}$ horizontal and $1 \mathrm{~mm}$ vertical displacement.

The FF permanent magnets are positioned such that they strengthen the magnetic field on the plasma side of the grid and weaken it on the beam side (fig 1).

\section{FF in the ITER reference design and in BATMAN}

In the ITER reference design the plasma grid between the beamlet groups consists of a thick copper plate [4]. The beamlet groups narrow the current cross-section, thus increasing the electric resistances for the PG current (fig 1). As a result the PG current flows mainly in between the beamlet groups and on the sides of the grid. By means of ANSYS FEM we evaluated the current density distribution in the PG. The results have been used as input for the QUICKFIELD 2D code to calculate the FF, since the PG current generates a magnetic field that is approximately $2 \mathrm{D}$, i.e. there is little variation in vertical 
direction, near the plasma grid. The BATMAN experiment [2] configuration (permanent magnets only) has also been calculated and compared with the ITER reference solution: 4kA DC PG current plus $30 \mathrm{~mm} \times 20 \mathrm{~mm} \mathrm{Sm-Co}$ permanent magnets on the sides of the source, originally developed for the arcdriven source [4].

Two main parameters have been considered in this analysis. One is the $B_{x}$, horizontal component of the magnetic field, calculated at different distances with respect to the $P G$ on the plasma side. The second is the $\int B_{x} d L$, integral of $B_{x}$ calculated along a line perpendicular to the PG, in $Z$ direction, between the PG and the source of fast electrons. In the RF source fast electrons come from the drivers, so the $\int B_{x} d L$ is calculated between the $P G$ and the ion source back plate, positioned at $220 \mathrm{~mm}$ from the PG.

From this preliminary study these considerations arise (see fig. 3 and 4):

- Absolute $B_{x}$ value of the BATMAN FF is significantly higher than in ITER reference FF.

- The $\int B_{x} d L$ calculated for an ITER-like RF source is much lower than the $\int \mathrm{B}_{\mathrm{x}} \mathrm{dL}$ for the BATMAN source. This is due to the fact that the width of the BATMAN source is much smaller than in an ITER-like source and therefore the permanent magnets are positioned closer to each other.

- As expected, the magnetic field at $2 \mathrm{~mm}$ from the PG surface, on the plasma side, has a considerable non-uniformity. Two kinds of "ripple" can be identified. The small ripple is due to the single apertures, where current can flow only on metal parts around the holes. A big 
ripple is then due to the fact that the current flows preferentially between the beamlet groups, where the grid is thick and no big electrical resistance is present. This non-uniformity on the magnetic FF is still present at 20 and $50 \mathrm{~mm}$ from the PG surface.
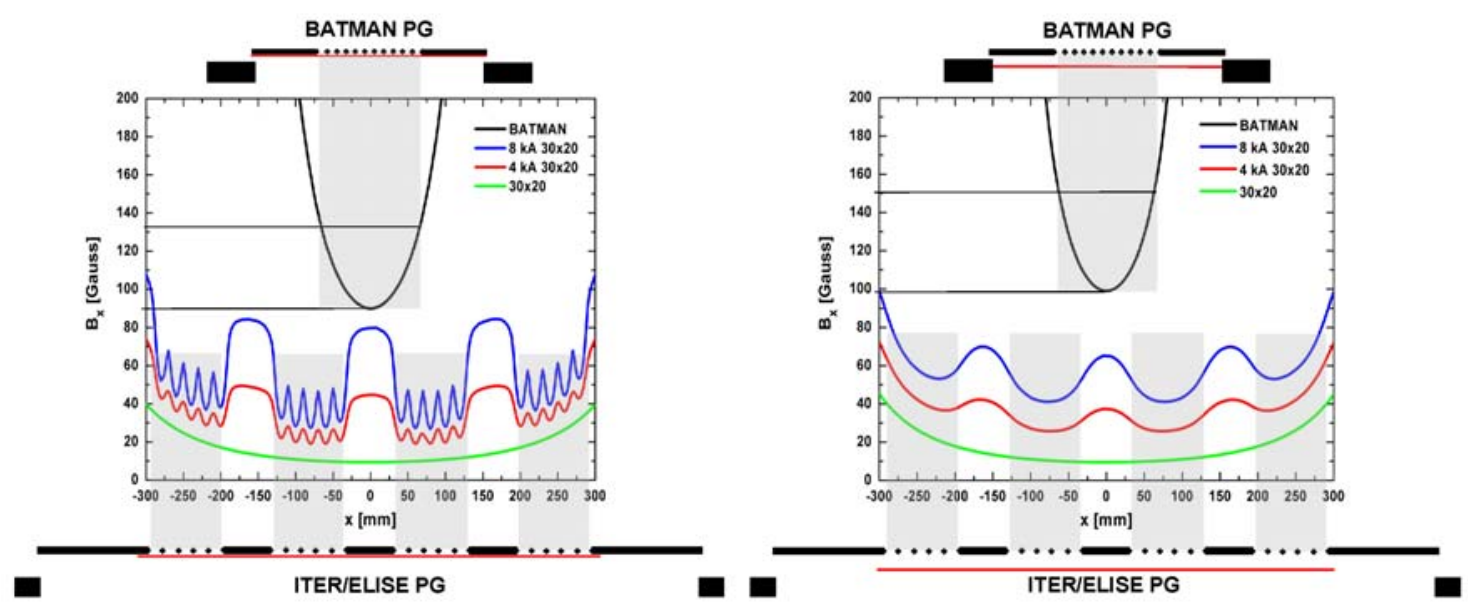

Figure 3 "Ripple" of the $B_{x}$ component of the FF across the extraction area of ELISE for different combinations of plasma grid current and permanent magnets; left: in a distance of $2 \mathrm{~mm}$ from the plasma grid, right: $20 \mathrm{~mm}$ from the plasma grid. Aperture positions indicated above and below graphs. For comparison, the $B_{x}$ field of BATMAN is plotted in each figure.

The FF of the ITER reference design differs considerably from the BATMAN FF, mainly due to the different sizes of the sources. 


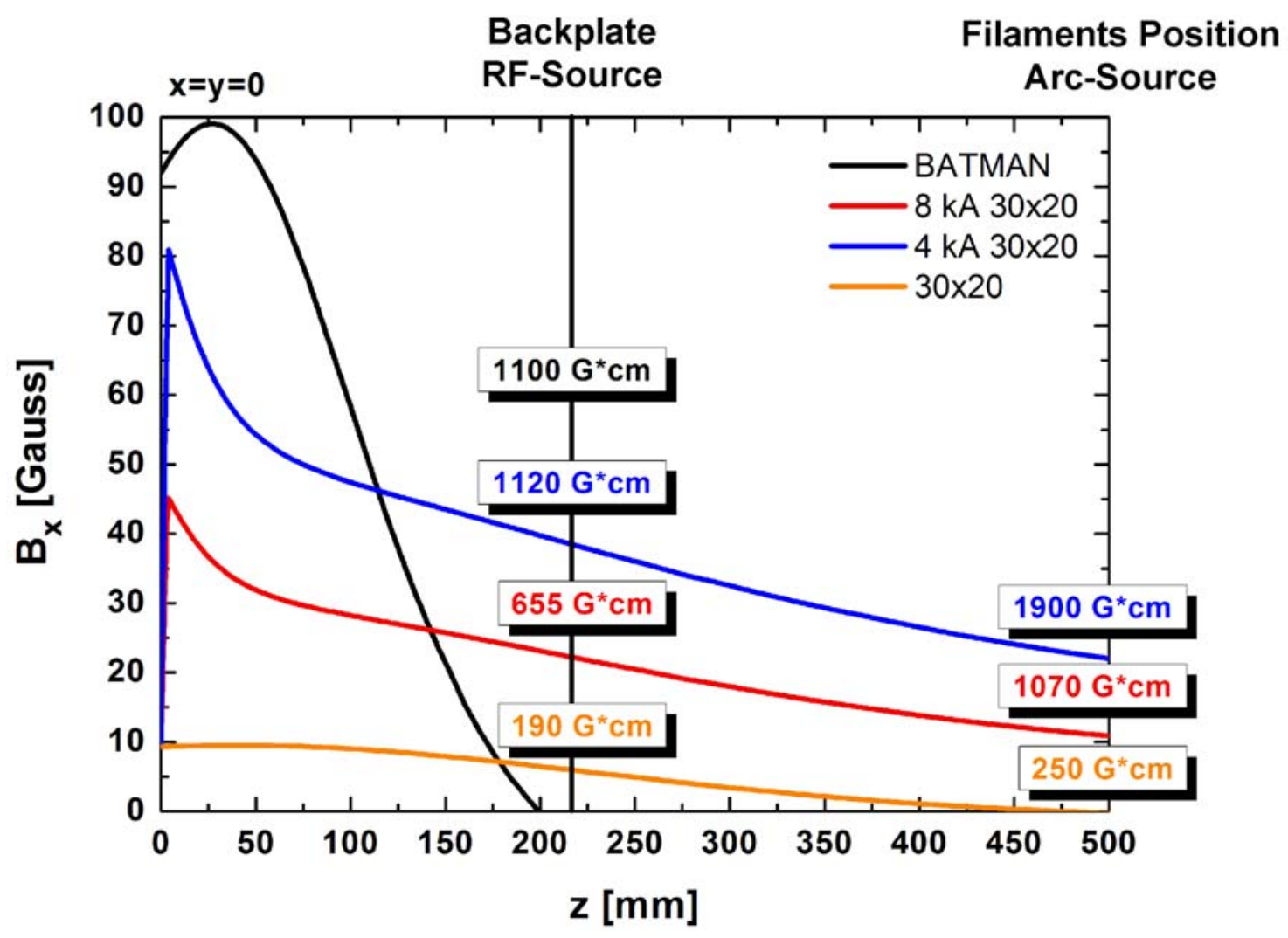

Figure $4 B_{x}$ filter field component for different combinations of permanent magnets and plasma grid current. Corresponding $\int \mathrm{B}_{x} \mathrm{dL}$ values have been calculated respect to the fast electron source: the back plate for the RF source and the filaments for the arc-driven source, respectively. (black = BATMAN, red $=$ ITER-like source with $8 \mathrm{kA} \mathrm{PG}$ current and permanent magnets, blue = ITERlike source with $4 \mathrm{kA}$ PG current and permanent magnets, yellow = permanent magnets only). (For interpretation of the references to color in the figure caption, the reader is referred to the web version of the article.)

The $\int B_{x} d L$ of the FF could be increased in ITER by raising the PG current, but this produces a higher field also in the accelerator region causing undesired deflection of accelerated particles and high power loads on the accelerator grids. In addition the ripples and non-uniformity of FF are increased. Up to now it is not clear which FF strength is necessary for the RF source, but it can be expected that a non-uniform FF with these large ripples affects the beam homogeneity. Therefore we put our research effort in reducing the FF nonhomogeneity in ELISE. 


\section{PG geometry for optimized magnetic FF}

The proposed solution for ELISE is to change the PG geometry such that the resulting current distribution reduces the non-uniformity in FF and generates a higher magnetic field in the central part of the PG. The PG geometry has been iteratively changed and optimized using ANSYS FEM code. A typical section of the ELISE PG has been modeled. The analyses consist of two steps.

- A model with thermal-electric conduction capability has been created in ANSYS to calculate the current density distribution. Ohmic heating, plasma load and water cooling have been taken into account to calculate the temperature distribution and the varying electrical resistivity.

- Permanent magnets and region around the PG have been modeled in ANSYS with magnetic elements and the current density distribution has been imported from the previous model.

Improvements in the FF uniformity have been obtained by changing the shape of the vertical manifolds of the cooling circuit of the PG and introducing pockets in the regions between the beamlet groups and on the sides of the PG (see fig 5). In addition ribs have been introduced on the beam side of the PG. This increases considerably the mechanical stiffness in the areas between the beamlet groups and on the sides of the PG without reducing significantly the electrical resistance. This design also gives the possibility to insert magnets in the pockets in a later stage of the experiment to test different FF configurations. As for the grids of the NBI systems of JET and ASDEX-Upgrade the manufacturing of the new PG design is possible by electro-deposition of copper. 
Cooling circuit manifolds
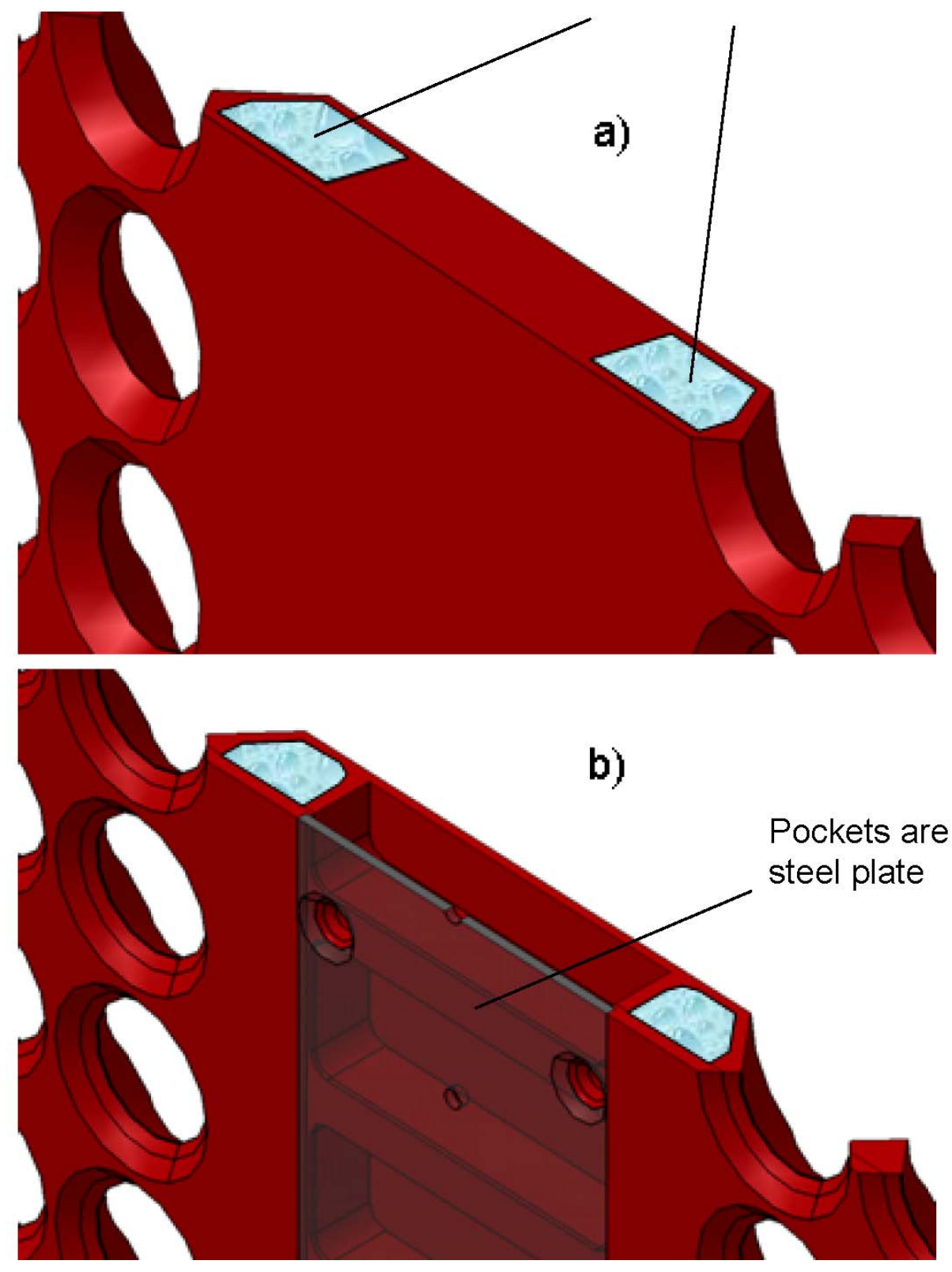

Figure 5 Section of different types of PG design. (a) Similar to the ITER reference PG. (b): ELISE PG with pockets to reduce the electric conduction between the apertures.

The results of the magnetic field calculations are shown in fig. 6 . It can be seen that the small ripple caused by the apertures is still present, but the big ripple has been reduced. The current flowing in the apertures region is now higher by $50 \%$ because more current is flowing in the center of the PG than in the 
previous design. Accordingly, the magnetic field in front of the PG is larger for the same total current. The minimum value of the magnetic field in front of the PG is increased by $50 \%$ and the big ripples in the magnetic field at $2 \mathrm{~mm}$ from the surface have been reduced. The non-uniformity in the FF almost disappears at $20 \mathrm{~mm}$ from the PG surface.

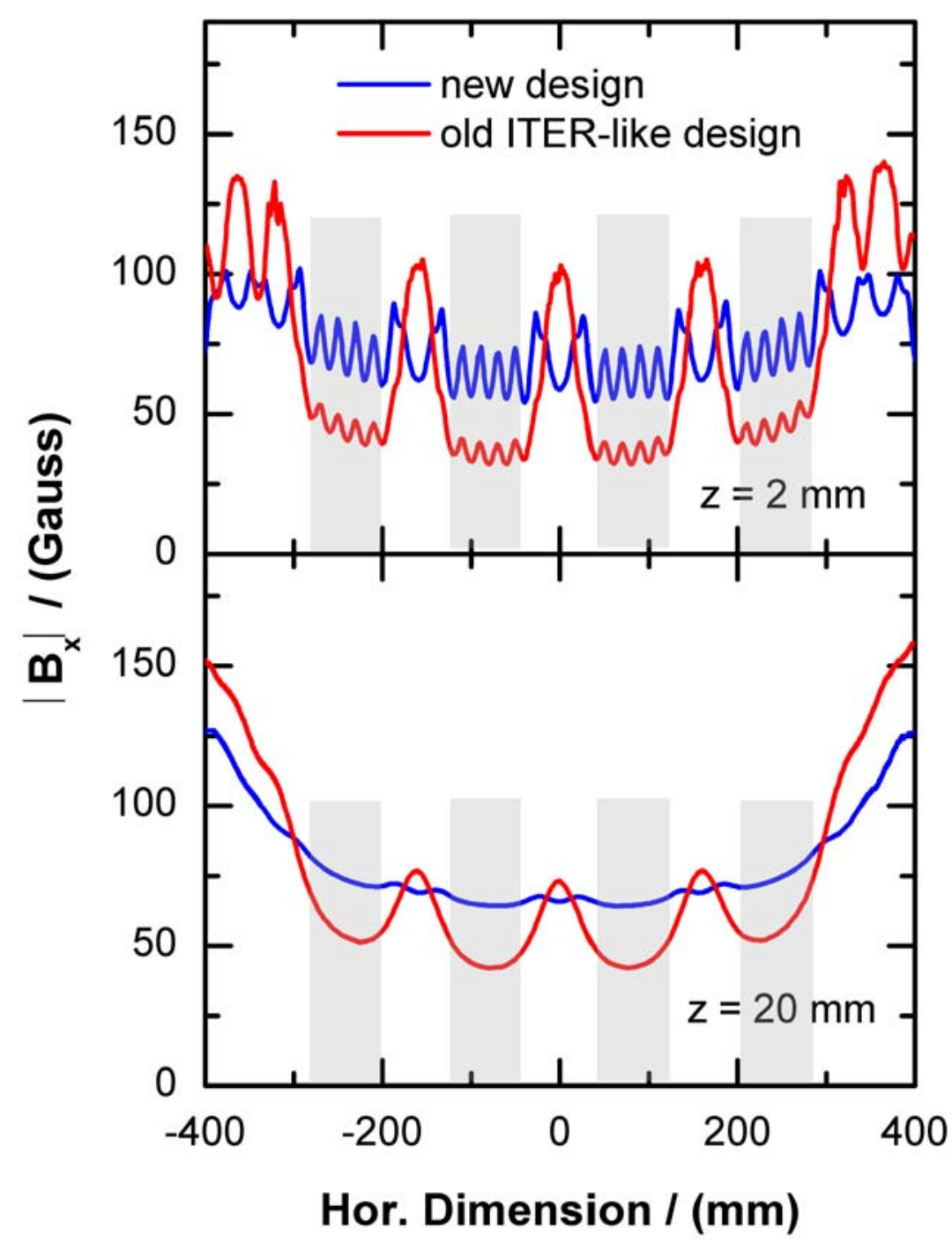

Figure 6 Filter field distribution across the extraction area of ELISE for the old and new plasma grid geometry in a distance of $2 \mathrm{~mm}$ and $20 \mathrm{~mm}$ from the plasma grid for $8 \mathrm{kA}$ current and permanent magnets. With the new plasma grid design, the field ripple is reduced and the field strength is increased.

The ${ }^{B} B_{x} d L$ has been calculated along different lines perpendicular to the $P G$,

both in the reference and in the new design. It can be seen that the new design 
produces a more uniform $\int \mathrm{B}_{\mathrm{x}} \mathrm{dL}$ distribution. In addition it is slightly higher in front of the extraction areas (see fig. 7).

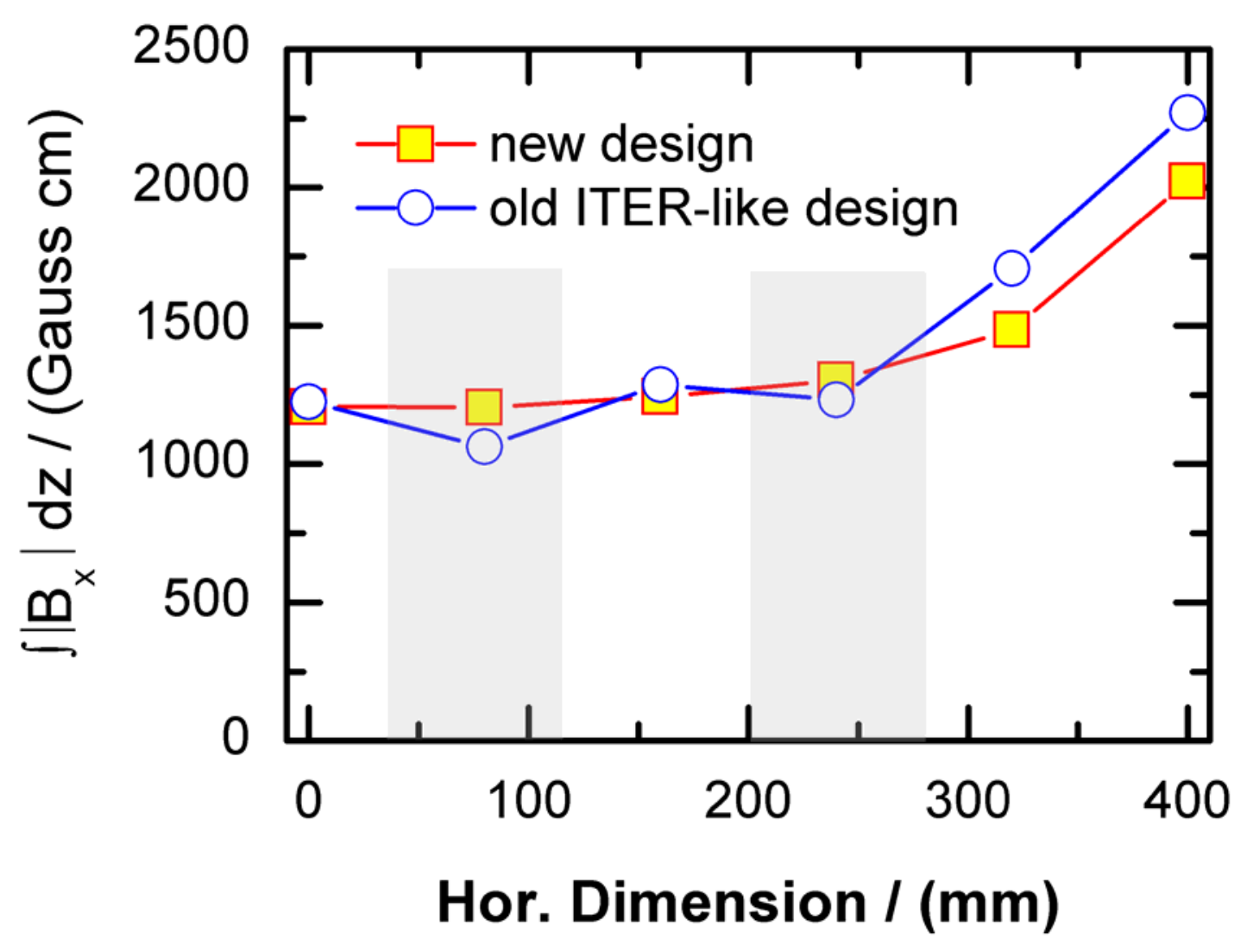

Figure $7 \int \mathrm{B}_{\mathrm{x}} \mathrm{dL}$ calculated for the $\mathrm{x}$ component of the magnetic field at the center of the beamlet groups and in between the beamlet groups for $8 \mathrm{kA}$ current and permanent magnets $(20 \mathrm{~mm} \times 30 \mathrm{~mm})$. The beamlet groups are indicated. It can be seen that with the old design there is a ripple on the $\int \mathrm{B}_{\mathrm{x}} \mathrm{dL}$ corresponding to the beamlet groups. This is not the case with the new design.

This means that with the new design a higher $\int \mathrm{B}_{\mathrm{x}} \mathrm{dL}$ can be generated in front of the beamlet groups, where a higher B field might be more important. As an alternative the $P G$ current could be reduced by $20 \%$ maintaining the same $\int B_{x} d L$ in front of the beamlet groups of the reference design.

\section{Electron suppression magnets (ESM) configurations}

Another magnetic field source in the extraction region are the electron suppression magnets (ESM) embedded in the EG. The ESM are required to 
deflect the co-extracted electrons. Although these magnets are not inside the source, the experience acquired at IPP suggests that these magnets influence drastically the plasma source performance [2]. In ELISE it will be possible to align the ESM both in horizontal and vertical direction, enabling investigations of the source performance with the ESM field parallel or orthogonal to the FF. In the first part of the study the role of the ESM has not been taken into account. In the second part of the study the influence of these magnets on the FF has been investigated as follows.

Two possible configurations have been considered. In the first one the ESM are oriented as dipoles vertically so that the generated magnetic field is parallel to the FF, alternating in horizontal direction. This will be called "parallel" orientation. This configuration has also been proposed for the ITER SINGAP accelerator design, but has never been used in large negative ion sources. The second configuration consists of ESM oriented as dipoles horizontally generating a magnetic field "orthogonal" to the FF. This option has been used in the BATMAN experiment and other experiments worldwide so far. The EG in ELISE allows investigation of both configurations.

The parallel and orthogonal configurations have been investigated with ANSYS, the results are shown in fig. 8 . It can be seen that in parallel orientation the ESM generate a magnetic field that is alternatively added and subtracted for each aperture column to the FF. This creates zero-field regions once every two apertures, could lead to a strong non-uniformity in filtering the electrons and is potentially dangerous. In fact, even if the overall power deposition on the EG is within the operational range, there could be a very localized heat load in 
correspondence to the apertures where the electron leakage is higher, leading to a localized melting of the EG.

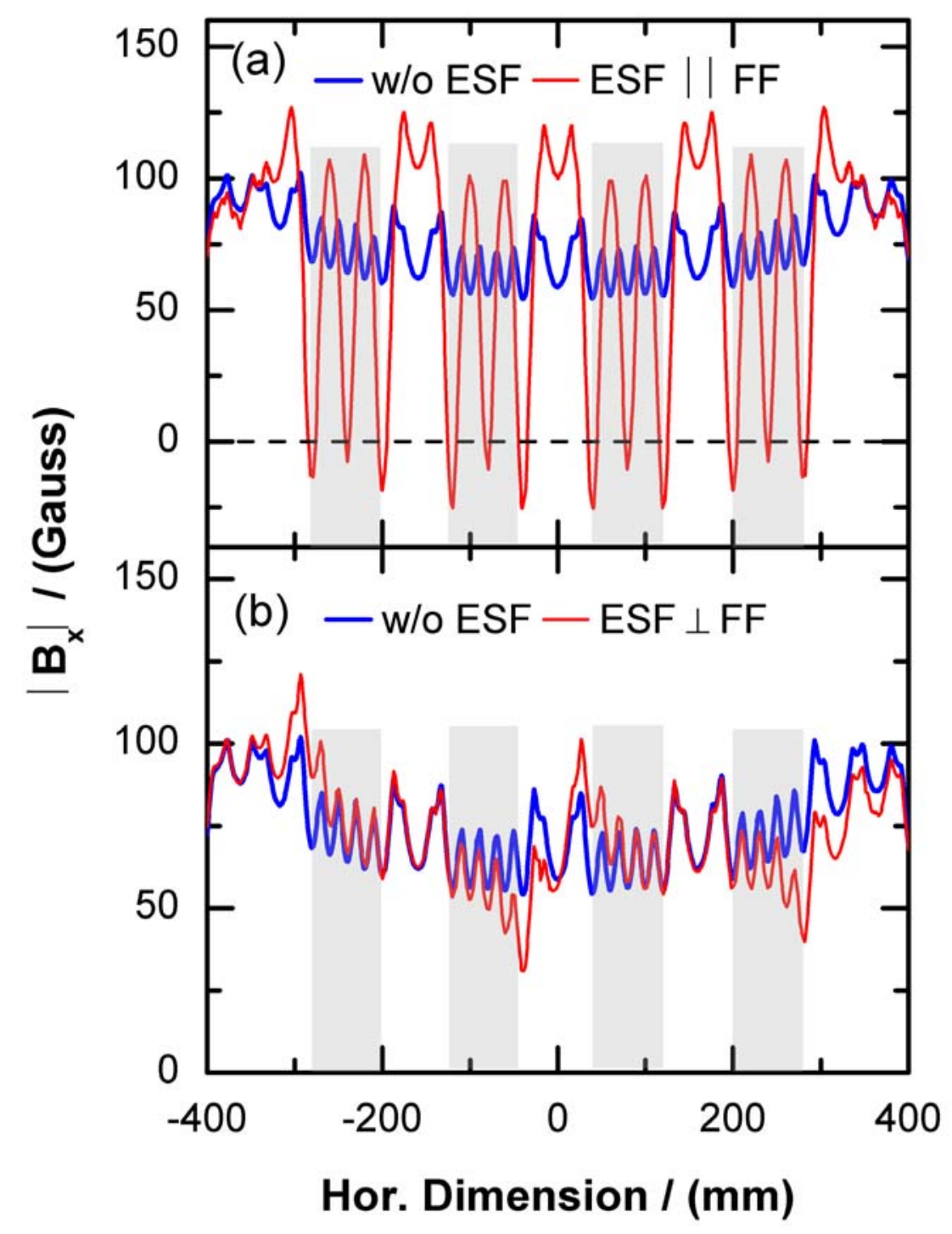

Figure 8 Effect of the orientation of the electron suppression field (ESF) on the $\mathrm{B}_{\mathrm{x}}$ component calculated at a distance of $2 \mathrm{~mm}$ from the plasma grid for $8 \mathrm{kA}$ current and permanent magnets. (a) Parallel configuration (vertical magnets). (b) Orthogonal configuration (horizontal magnets).

The orthogonal orientation of the ESM on the contrary generates a much more uniform magnetic field in front of the PG. Due to the design of the cooling system of the EG it is not possible to realize horizontal magnet grooves straight 
through the whole width of the grid [1]. Small magnetic field distortions due to gaps without magnets are visible from the FF calculation, but they can be considered acceptable in terms of FF homogeneity.

\section{Further improvements}

Although the new PG design offers a significant improvement of the FF homogeneity, the FF in front of the PG, with 4kA of PG current, is still lower than in the BATMAN configuration. The PG current needed to reach the $\int B_{x} d L$ of the BATMAN experiment is still higher than the $4 \mathrm{kA}$ foreseen in the ITER reference design, even with the improved PG geometry. Also FF gradients are different with consequences for the source performance that can not be estimated up to now.

Several solutions are possible but have to be assessed, like magnets inserted in the pockets or in front of the PG in the regions between the beamlet groups, iron jokes, coils inside or outside the source.

\section{Summary}

The ITER reference FF, originally developed for the arc-driven source, has been compared with the successful BATMAN RF source experiment and other configurations. The aim was to design a plasma grid and a magnetic configuration for an ITER-like RF source, the ELISE experiment, capable of producing a homogeneous FF with the same relevant parameters $\left(B_{x}\right.$ and $\int \mathrm{B}_{\mathrm{x}} \mathrm{dL}$ ) of the existing negative ion sources. Major modifications have been introduced in the design of the PG such that the FF is more homogeneous, stronger by $50 \%$ in front of the $P G$ and the integral $\int B_{x} d L$ is higher in front of the 
beamlet groups than in the ITER reference design for the same total plasma grid current.

The absolute value of the FF in front of the PG is still lower than in the BATMAN source. To increase this value and possibly reduce the PG current other solutions have to be investigated. The new design leaves room for positioning magnets in the PG or on the sides of the extraction areas.

The ESM have also been considered for their influence on the magnetic field in the extraction area. Parallel and orthogonal orientations have been calculated and compared, providing data that support the latter solution.

\section{Acknowledgments}

This work was supported by a grant (03FUS0002) from the German Bundesministerium fuer Bildung und Forschung as well as by a grant (TW6THHN-RSFD4) from the European Union within a framework of EFDA (European Fusion Development Agreement). This support is gratefully acknowledged.

The authors are solely responsible for the content.

The author would like to acknowledge the contribution of Dr. Piero Agostinetti from Corsorzio RFX, Padova 


\section{References}

[1] B. Heinemann, et al., Design of the "half-size" ITER neutral beam source for the test facility ELISE, SOFT conference, Fusion Eng. Des. (2008).

[2] Speth E. et al, Overview of the RF source development programme at IPP Garching, Nuclear Fusion 46 (2006), S220

[3] M. Froeschle et al., Fusion Eng. Des. 82 (2007) 887-896

[4] ITER Technical Basis, Design Description Document, Section 5.3 (DDD5.3), International Atomic Energy Agency, 2001.

[5] A. Stäbler, et al., Development of a RF-driven ion source for the ITER NBI system, SOFT conference 2008, Fusion Eng. Des. (2008)

[6] Franzen P. et al, Physical and experimental background of the design of the ELISE test facility, Proceedings of the 1st International Conference on Negative lons, Beams and Sources (NIBS), Sept. 2008 